\title{
Incertidumbre financiera de las Empresas Sociales del Estado en Colombia: análisis exploratorio para los años 2010 - 2015
}

\author{
Arboleda Posada, Gladys Irene ${ }^{1}$; García Arango, David Alberto ${ }^{2(*)}$; Henao Villa, Cesar Felipe ${ }^{2}$ \\ ${ }^{1}$ Universidad de Antioquia, Facultad de Medicina, Medellín, Colombia. \\ ${ }^{2}$ Corporación Universitaria Americana, Facultad de Ingeniería, Medellín, Colombia.
}

\section{RESUMEN}

Las Empresas Sociales del Estado (ESE), enfrentan el desafío de manejar mejor sus recursos, para lo cual deben utilizar técnicas contables y de análisis financiero actualizadas, como lo sugieren los principios y prácticas financieras. El presente artículo pretende analizar los problemas administrativos de índole financiero, especialmente los relacionados con la liquidez, rentabilidad y endeudamiento que se desprenden de contratar la prestación de servicios de salud con las empresas prestadoras y los municipios en la región central de Colombia. A nivel metodológico se desarrolla un estudio descriptivo de corte transversal, los datos fueron tomados de un archivo suministrado por el Ministerio de Protección Social que contiene un total de 3226 registros de Empresas Sociales del Estado para los años 2010 a 2015, distribuidas en 12 departamentos para un total de 547 instituciones por niveles de atención. Se obtienen los resultados de aplicación de un análisis factorial exploratorio y su consecuente modelo de regresión, dando como resultado una relación entre las cuentas por cobrar y los indicadores de liquidez, endeudamiento y rentabilidad. Como conclusión se identifica la necesidad que las Empresas Sociales del Estado tengan claridad sobre su situación financiera, en términos de liquidez, endeudamiento y rentabilidad, para poder cumplir con su objetivo social de preservar la salud de la población, prestando servicios de calidad.

Palabras clave: Administración Financiera de Hospitales. Costo de Enfermedad. Costos y Análisis de Costo. Seguridad Social. Apoyo Financiero. Incertidumbre.

\section{Financial uncertainty of State's Social Enterprises in Colombia: exploratory analysis for the years 2010 - 2015}

\section{ABSTRACT}

The State's Social Enterprises face the challenge of better managing their resources, for which they must use updated accounting and financial analysis techniques, as suggested by financial principles and practices. The objective of this paper is to analyze the administrative problems of a financial nature, especially those related to liquidity, profitability and indebtedness that arise from contracting the provision of health services with provider companies and municipalities in the central region of Colombia. As methodology, a descriptive cross-sectional study is developed. The data was been taken from a file provided by the Ministry of Social Protection that contains a total of 3,226 records of State Social Enterprises for the years 2010 to 2015 , distributed in 12 departments for a total of 547 institutions by levels of care. The above results in the application of an exploratory factor analysis whose could predict a relationship between accounts receivable and indicators of liquidity, indebtedness, and profitability. The need of this study is to identify variables related to financial situation of this enterprises, in terms of liquidity, indebtedness and profitability, in order to meet their social objective of preserving the health of the population, providing quality services.

Keywords: Financial Management. Hospital Cost of Illness. Costs and Cost Analysis. Social Security. Financial Support. Uncertainty.

Recibido: 02/05/2021 Aceptado:01/06/2021

Correspondencia: (*) dagarcia@coruniamericana.edu.co 


\section{INTRODUCCIÓN}

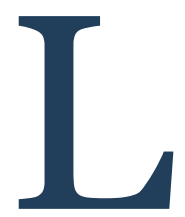

as circunstancias y realidades que se presentan en Colombia y particularmente aquellas que inciden de manera directa en los actores que intervienen en el sector de la salud, plantean la imperiosa necesidad de analizar las diferentes instituciones de salud, especialmente las Empresas Sociales del Estado (ESE) desde el punto de vista financiero.

No escapa a la percepción de ningún analista la trascendencia de variables como la cartera, la liquidez, la rentabilidad y endeudamiento entre otras, y su incidencia directa como determinante de la viabilidad y de la estabilidad de las empresas en el futuro.

Como es bien sabido por los administradores y por otros profesionales, el análisis financiero proporciona información de alto valor para el proceso de toma de decisiones y la elaboración de indicadores. La solidez de estos se convierte en la carta de navegación confiable para las empresas; por ende, la falta de esos indicadores o la fragilidad de estos puede llevar al fracaso.

Las principales circunstancias que motivaron la realización del presente artículo, fueron las inconsistencias observadas en las últimas crisis hospitalarias como la del 2015(Suárez-Rozo et al., 2017), que no es un hecho aislado, pues se ha repetido, hasta llegar al punto de ir en detrimento de la salud financiera de las ESE y otros campos desde 1993.

Actualmente, los principales problemas de los hospitales colombianos se encuentran en el área financiera (especialmente lo que tiene que ver con la liquidez) y se originan en el inadecuado sistema de contratación imperante, a todas luces asimétrico; además, como si lo anterior fuera poco, los aseguradores no giran los dineros de forma oportuna o simplemente no los giran, con lo cual someten a los hospitales a absorber altos costos o incluso a trabajar a pérdida o en el límite de la relación. En ese sentido, en el año 2015, el 57\% de las ESE registraron altas cifras en su renglón de cartera morosa o de difícil cobro, lo cual afecta la posibilidad de brindar más y mejor atención a la población.

El problema principal radica en que "además de las restricciones presupuestales de política económica, el flujo de recursos impacta la prestación y calidad de los servicios de salud. Ejemplo, para el Régimen Subsidiado, entre el momento en que se giran los recursos de alguna fuente de financiación (SGP, Fosyga, Rentas Cedidas, Regalías, ETESA, CCF) y son registradas por la cuenta maestra del municipio $y$ luego transferida a las EPS, transcurren unos 60 días, y para que las EPS les giren a las IPS pasan 30 días. En total, la mora toma aproximadamente 90 días".

Con base en lo anterior, el artículo presenta el análisis realizado a las cuentas por cobrar esbozando las dificultades que este tema trae a colación en la región andina en Colombia.

\section{MARCO TEÓRICO}

El cumplimiento de obligaciones no solo determina la buena salud general de una empresa, sino las buenas condiciones de sus trabajadores, proveedores y demás grupos de interés. La morosidad de las Empresas Prestadoras de Servicios de Salud (EPS) del régimen contributivo y subsidiado va en detrimento de la imagen que la comunidad se forma de la entidad; además; dicho incumplimiento genera desfases en los indicadores financieros, lo que causa incertidumbre precedente a las crisis. Estas situaciones y la gran crisis del 2015 (Ochoa \& Moctezuma, 2012), de la que aún se sienten con fuerza los coletazos, no deja de ser importante a la hora de analizar tales empresas desde el punto de vista financiero. Para profundizar en el estudio de dicha situación se obtuvieron indicadores de liquidez, rentabilidad y endeudamiento, con el fin de describirlos y compararlos con la cartera, situación apremiante al momento de examinar dichas instituciones; este análisis presentará un diagnóstico financiero de las ESE. Con los indicadores mencionados será posible tomar decisiones de planeación y para que el proceso de control se vea favorecido.

Hasta antes de la reforma introducida por la ley 100 
en 1993 (Por la cual se crea el sistema de seguridad social integral y se dictan otras disposiciones, 1993), los hospitales públicos tenían una forma particular de consecución y manejo de sus recursos financieros, pues su ejercicio se daba en el marco del principio de subsidiar la oferta. Con la reforma, el principio se invirtió y los subsidios se destinaron a la demanda; es decir, se pasó de un modelo altamente protegido por el Estado, a un modelo de libre competencia en el mercado (autofinanciado).

Así las cosas, se aceleró el proceso de descentralización, lo cual implicó que los hospitales se transformaran en empresas de naturaleza estatal (nacimiento de las ESE), con lo cual se vieron en la obligación de conseguir sus recursos a través de sus propias operaciones y al mismo tiempo, adoptar el sistema financiero y contable sujeto a normas nacionales e internacionales. En el ámbito interno, los hospitales convertidos en ESE se vieron obligados a adoptar sistemas o programas de calidad, de costos, de facturación y en general, adoptar procesos gerenciales fuertemente influenciados por preceptos de la empresa privada (Arango et al., 2010)

Lo anterior ha obligado a trabajar sobre metas y resultados: producción y mejoramiento de la calidad de los servicios, sostenibilidad financiera y planes de ventas, eficiencia y compromisos con los planes nacionales, departamentales y municipales entre otros, es decir, ahora dependen de su propio esfuerzo para ser rentables, sin una protección directa del gobierno, cuestión que las ha abocado (por las mismas dificultades que se presentan con el sistema), a la generación de crisis que tienen en vilo su supervivencia (Rodríguez, 2012b).

El sistema se ha complicado aún más con la aparición de figuras como las entidades aseguradoras (EPS), pues son ellas las que de manera primaria captan los recursos financieros, con el consecuente desbalance frente a los demás actores, si a lo anterior se suma la carencia de oportunidad en el giro de los dineros por parte de las EPS hacia los hospitales, el panorama se torna aún más oscuro (Evaluación del Sistema General de Seguridad Social en Salud y del Sistema General de Riesgos Profesionales durante los últimos 10 años, 2004)
La figura de los intermediarios en el sistema de salud en Colombia es la que lo contamina, privándolo de una estabilidad que asegure la sostenibilidad financiera de las ESE como de las EPS, siendo este un factor determinante para propiciar las crisis hospitalarias, en donde el principal afectado es el ciudadano perteneciente a poblaciones vulnerables que por los determinantes de la salud, utiliza con más frecuencia dichos servicios; esta inestabilidad y la vulnerabilidad de las ESE que depende mayormente de los recursos que adquieren por prestación de servicios son una situación interminable, siendo factor propiciador de dicho desbalance, la morosidad de las EPS para pagar los servicios contratados con las IPS (Cárdenas \& Velasco, 2014).

Como parte de las estrategias para el mejoramiento de la gestión en el marco de la sostenibilidad, algunos autores identifican la importancia del planteamiento de los Objetivos de Desarrollo Sostenible como una estrategia que ha aportado en tanto que "la declaración de los ODS ha proporcionado un entorno propicio para implementar legislaciones necesarias, reformar la organización del financiamiento de la salud y revisiones de las políticas nacionales de salud para alinearlas con el compromiso del país con la cobertura universal de salud" (Odoch et al., 2021, p. 1).

Según Kutzin (2013); Mclntyre \& Kutzin (2016), la cobertura universal de salud se ve altamente impactada debido a los efectos de los intermediarios en aspectos de transparencia, rendición de cuentas, eficiencia y equidad. Estos aspectos deben ser revisados con la finalidad del establecimiento de políticas orientadas hacia el cumplimiento de los ODS. En ese sentido, un aspecto recomendable es el desarrollo de políticas uniformes y procedimientos legislativos que posibiliten la eliminación del impacto negativo de la privatización en la educación, la investigación y los servicios de salud(Kuwaititi \& Al Muhanna, 2020), en ese sentido, los entes de control juegan un papel fundamental.

Respecto a estrategias orientadas a la ampliación de cobertura, mejoramiento del servicio y sostenibilidad de los sistemas de salud, la literatura al 
respecto presenta entre otras opciones la consideración de programas de paramédicos en comunidades rurales y urbanas como soporte a la gestión de las ESE, en ese sentido, los estudios realizados identifican la factibilidad a nivel de costo-efectividad donde es ideal la consideración de un marco metodológico transformador para la sostenibilidad que podría ser extrapolado a los diferentes programas propuestos en las ESE para la optimización de la operatividad. (Huang et al., 2018). Adicionalmente, se identificó la aplicación de la gerencia de valor que según Rojas(2016), tiene el objetivo de "conservar en términos de saneamiento fiscal, estabilidad financiera, un adecuado control y manejo del presupuesto, las finanzas y el patrimonio público" (pág. 173) esto se concibe como un gran paso para introducir conceptos como los de la "Nueva gestión Pública" que "fundamenta la creación de una administración eficiente y eficaz, que cubra las necesidades de los ciudadanos a bajo costo posible, con servicios competitivos, que promuevan servicios de calidad, aplicando sistemas de control y transparencia que favorezcan la participación ciudadana" García (2007) citado en Jiménez Sánchez, Rojas Restrepo, \& Ramírez Tapias (2017).

\section{METODOLOGÍA}

Para el desarrollo de la investigación se consideró un análisis descriptivo de corte transversal, la lógica de interpretación de los resultados de investigación, se adecúan al paradigma del pragmatismo en el marco del razonamiento abductivo propuesto por Samaja(2012), según el cual, los resultados y sus interrelaciones se adecúan a los presupuestos teóricos para generar la obtención del caso. Los datos hacen parte de un archivo suministrado por el Ministerio de Protección Social que contiene información financiera de un total de 3226 registros de Empresas Sociales del Estado para los años 2010 a 2015, en la región Andina para los departamentos de Santander, Tolima, Norte de Santander, Risaralda, Quindío, Córdoba, Antioquia, Huila, Cundinamarca, Caldas, Boyacá, Bogotá.

Se analizaron los datos de un total de 547 instituciones por niveles de atención. La técnica de análisis de datos se aplicó en el software SPSS versión 23
(IBM SPSS Statistics for Windows, Version 23.0, 2015) realizando un análisis factorial exploratorio y su consecuente análisis de clúster jerárquico. La complementariedad de estas técnicas de análisis de datos se puede ver en Gorman y Primavera(1983), bajo esta estructura, la lógica de desarrollo del análisis consta de dos etapas:

Etapa I: Aplicación de un análisis factorial donde se identifican matrices de correlación y se clasifican los indicadores financieros (liquidez, endeudamiento y rentabilidad) dando como resultado componentes y subcategorías de análisis mediante las comunalidades obtenidas.

Etapa II: Se establecen conclusiones a partir de la información obtenida y se proponen futuras líneas de investigación.

\section{RESULTADOS}

En la aplicación del análisis factorial, se identificaron componentes que relacionan las variables en cuestión para los años 2010-2015 en la región andina. Los componentes son grupos de variables que según los datos obtenidos tienen similitudes estadísticamente significativas. En la Tabla 1 se presentan los componentes obtenidos por año y para cada indicador de Liquidez, Endeudamiento y Rentabilidad. Se identifica, por ejemplo, que el capital neto de trabajo, el EBITDA y el ROA, hacen parte de un mismo factor que intervino significativamente para el año 2010 puesto que tienen el mismo indicador (C21).

De la Tabla 1 se pueden identificar algunas variables que no tienen clasificación en los componentes para algunos de los años, esto es debido a que la inclusión de éstas en el análisis conlleva una matriz no definida positiva, con lo cual se tiene que las variables son dependientes, al excluir la variable del análisis se tienen variables independientes. Respecto a los elementos de la Tabla 1 , se identifica que Cij representa el componente i para el indicador $\mathrm{j}$, de esta forma $\mathrm{i}=1,2,3$ para $\mathrm{j}=$ Liquidez, Endeudamiento, Rentabilidad. Es importante considerar que para la pertenencia a uno de los componentes se tuvo en cuenta valores obtenidos de rotación varimax superiores a 0,4 y teniendo en 
cuenta una prueba de esfericidad significativa garantizando de esta forma una matriz cierta positiva. Los análisis para cada indicador se presentan como sigue.

\section{Análisis para el indicador de liquidez:}

Se observa que el índice de rotación de cartera corriente y los días de rotación de cartera corriente tienen una relación inversa, lo cual es consecuente con la misma definición que proponen. El componente en el cual se encuentran estas dos variables es independiente de otras variables para los años 2010, 2012 y 2014. Para los años 2011 y 2015 se observa una interacción con la variable ROA. Para el año 2013, el componente del índice de rotación de cartera corriente y los días de rotación de cartera corriente también contiene el capital neto de trabajo, EBITDA y ROA.

Respecto a las variables de razón corriente, razón rápida o prueba ácida y la prueba de extrema liquidez - corriente se encuentran en el mismo componente independiente del año, vale la pena resaltar que algunas veces no aparecen las variables en los componentes. Específicamente la prueba de extrema liquidez - corriente no se encuentra en los componentes para los años 2010, 2012, 2013 y 2014, esto se puede comprender debido a que la variabilidad de este valor es explicada por la variabilidad de los otros elementos del componente. En cuanto al capital neto de trabajo, es importante identificar que, para los años en análisis, éste está en el mismo componente que el EBITDA.

\section{Análisis para el indicador de Endeudamiento:}

En este indicador, los años 2010, 2011, 2013 y 2015 se comportan de la misma manera en sus componentes donde el componente $\mathrm{C} 1$ se forma con el índice de endeudamiento, leverage o apalancamiento y es inverso a la solvencia o respaldo total. El componente $\mathrm{C} 2$ se forma únicamente con la concentración del endeudamiento en el corto plazo. Para los años 2012 y 2014, la variación la dan el Leverage o apalancamiento para el año 2012 así como el índice de endeudamiento (no existente como componente), el Leverage o apalancamiento y la solvencia o respaldo total para el año 2014.

\section{Análisis para el indicador de Rentabilidad:}

En este indicador, los años 2010, 2011, 2013, 2014 y 2015 tienen el mismo comportamiento por componentes donde el componente $\mathrm{C} 1$ se forma con el margen de utilidad sobre las ventas, la rentabilidad del patrimonio y la rentabilidad del activo, el componente C2 está conformado únicamente por el margen bruto. Respecto al año 2012 se identifica que el margen bruto pasa para el componente 1 y la rentabilidad del patrimonio pasa para el componente 2 . Vale la pena considerar que la rentabilidad sobre el patrimonio no logró pertenencia a los componentes, lo cual se interpreta como que la variabilidad de este ítem ya está representada por todos los demás.

Se observan como variables especialmente fuertes, la prueba de externa liquidez-corriente para el año 2011; concentración del endeudamiento en el corto plazo para los años 2010, 2011, 2013, 2014 y 2015; margen bruto para los años 2010, 2011, 2013, 2014 y 2015; rentabilidad del patrimonio para los años 2012. Respecto a los indicadores de endeudamiento y rentabilidad, se encuentra que los años 2010, 2011, 2013 y 2015 son similares. El indicador de liquidez es el que más variedad de componentes presenta, de ahí que pueda considerarse que la liquidez es más sensible a las diferentes condiciones de los períodos analizados. 
Tabla 1. Relación de variables según indicador y componente para los años 2010-2015. C1, C2 y C3 representan componentes que son diferentes dependiendo del indicador.

\begin{tabular}{|c|c|c|c|c|c|c|c|}
\hline Indicador & Variable asociada & 2010 & 2011 & 2012 & 2013 & 2014 & 2015 \\
\hline \multirow[t]{8}{*}{ Liquidez } & Índice de Rotación de cartera corriente & $\mathrm{C} 31$ & $-\mathrm{C} 21$ & $-\mathrm{C} 31$ & $-\mathrm{C} 11$ & $-\mathrm{C} 21$ & $-\mathrm{C} 31$ \\
\hline & Días de Rotación de cartera corriente & $-\mathrm{C} 31$ & $\mathrm{C} 21$ & C31 & $\mathrm{C} 11$ & $\mathrm{C} 21$ & C31 \\
\hline & Razón corriente & $\mathrm{C} 11$ & No & $\mathrm{C} 11$ & $\mathrm{C} 21$ & $\mathrm{C} 31$ & $\mathrm{C} 11$ \\
\hline & Razón rápida o prueba ácida & C11 & No & C11 & $\mathrm{C} 21$ & No & $\mathrm{C} 11$ \\
\hline & Prueba de extrema liquidez -corriente & No & $\mathrm{C} 31$ & No & No & No & $\mathrm{C} 11$ \\
\hline & Capital neto de trabajo & $\mathrm{C} 21$ & $\mathrm{C} 11$ & $\mathrm{C} 21$ & $\mathrm{C} 11$ & C11 & $\mathrm{C} 21$ \\
\hline & EBITDA & $\mathrm{C} 21$ & C11 & $\mathrm{C} 21$ & C11 & C11 & $\mathrm{C} 21$ \\
\hline & ROA & $\mathrm{C} 21$ & $\mathrm{C} 21$ & $\mathrm{C} 21$ & $\mathrm{C} 11$ & $\mathrm{C} 31$ & $\mathrm{C} 31$ \\
\hline \multirow[t]{4}{*}{ Endeudamiento } & Índice de Endeudamiento & $\mathrm{C} 12$ & $\mathrm{C} 12$ & $\mathrm{C} 12$ & $\mathrm{C} 12$ & No & $\mathrm{C} 12$ \\
\hline & Leverage o apalancamiento & $\mathrm{C} 12$ & $\mathrm{C} 12$ & $-\mathrm{C} 22$ & $\mathrm{C} 12$ & $-\mathrm{C} 12$ & $\mathrm{C} 12$ \\
\hline & Concentración del Endeudamiento en el Corto Plazo & $\mathrm{C} 22$ & $\mathrm{C} 22$ & $\mathrm{C} 22$ & $\mathrm{C} 22$ & $\mathrm{C} 22$ & $\mathrm{C} 22$ \\
\hline & Solvencia o respaldo total & $-\mathrm{C} 12$ & $-\mathrm{C} 12$ & $-\mathrm{C} 12$ & $-\mathrm{C} 12$ & $\mathrm{C} 12$ & $-\mathrm{C} 12$ \\
\hline \multirow[t]{5}{*}{ Rentabilidad } & Margen bruto & $\mathrm{C} 23$ & $\mathrm{C} 23$ & $\mathrm{C} 13$ & $\mathrm{C} 23$ & $\mathrm{C} 23$ & $\mathrm{C} 23$ \\
\hline & Margen de utilidad sobre las ventas & $\mathrm{C} 13$ & $\mathrm{C} 13$ & $\mathrm{C} 13$ & $\mathrm{C} 13$ & $\mathrm{C} 1$ & $\mathrm{C} 13$ \\
\hline & Rendimiento sobre el patrimonio & No & No & No & No & No & No \\
\hline & Rentabilidad del patrimonio & $\mathrm{C} 13$ & $\mathrm{C} 13$ & $\mathrm{C} 23$ & $\mathrm{C} 13$ & $\mathrm{C} 13$ & $\mathrm{C} 13$ \\
\hline & Rentabilidad del active & $\mathrm{C} 13$ & $\mathrm{C} 13$ & $\mathrm{C} 13$ & $\mathrm{C} 13$ & $\mathrm{C} 13$ & $\mathrm{C} 13$ \\
\hline
\end{tabular}

\section{CONCLUSIONES}

Con la referencia de que las cuentas por cobrar son el eje sobre el cual gira la liquidez de la empresa, y componente principal del flujo del efectivo es necesario que se realice una administración efectiva de la cartera en todas las instituciones, este proceso incluye el registro, clasificación de las cuentas por antigüedad y categorías como son corriente, vencida y cuentas de difícil cobro que regularmente se maneja con plazos de 1 a 30 dias, 30 a 60,60 a 90 y más de 90 días, situación que está lejos de cumplirse para el sector salud, donde en la mayoría de los casos está ya ha superado los 180 días.

La cartera y el inventario son los componentes básicos del Activo Corriente, en Términos reales son considerados como cuentas relativamente liquidas. Debido a que no se puede contar con la certeza de la oportunidad de traducirse en dinero en efectivo, lo que con frecuencia genera parala empresa periodos de incertidumbre, por lo tanto, determinar qué porcentaje corresponde a cartera ayuda a determinar si hay un exceso, generando lo que se denomina "fondos ociosos", los cuales tienen un alto costo de oportunidad y financiación.

Dar el incentivo de utilidad a los prestadores de servicios de salud sin la adecuada supervisión y control de entes reguladores (Rodríguez, 2012a) ha sido el insumo de la insostenibilidad financiera del sistema(García-Ubaque et al., 2012). El fenómeno financiero de la integración vertical, el uso indebido de recursos públicos, los dudosos recobros de las EPS al Fosyga; además de múltiples casos en los que se evidencia la muerte de pacientes debido a la pésima calidad del servicio de un problema social caracterizado por su crisis en variables estructurales(Rivera, 2011); (Morales \& García, 2017).

En conclusión, las situaciones plateadas anteriormente han generado una incertidumbre financiera para todo el sector salud, ocasionando situaciones de riesgo financiero para las empresas sociales del estado, por el requerimiento permanente de efectivo que estas requieren, lo cual no permite cumplir con el objetivo social preservar la salud de la población, prestando servicios de calidad (Santos et al., 2016).

Si bien es cierto que el panorama es complejo hay opciones en materia de gestión, el marco propuesto por (Huang, Ma, Sabljak, \& Puhala(2018), identifica la evaluación de necesidades de salud locales y de la comunidad para en un primer momento definir metas y objetivos de los programas, los cuales deberán estar articulados a proyectos estratégicos, desarrollar un portafolio de servicios detallado, desarrollar planes de entrenamiento, diseñar un plan de evaluación del programa, divulgación del programa, implementación, recolec- 
ción de datos, buscar soporte administrativo, legal y potenciales colaboradores, todo lo anterior debería estar asociado a sus respectivos componentes e indicadores a nivel financiero contrastando los niveles obtenidos en los estudios de factibilidad con los resultados de ejecución por intervalos similares de tiempo. De esta forma, se genera una gestión más articulada con las necesidades locales y en articulación con los factores económicos y los objetivos estratégicos a largo, mediano y corto plazo. 


\section{REFERENCIAS}

Arango, L., Rodríguez, C., \& López, R. (2010). Cálculo de los costos de calidad y no calidad en empresas de salud y creación de un sistema de medición. CIFE, 12 (16), 65-78.

Cárdenas, M., \& Velasco, B. M. (2014). Incidencia de la morosidad de las cuentas por cobrar en la rentabilidad y la liquidez: estudio de caso de una Empresa Social del Estado prestadora de servicios de salud. Rev. Fac. Nac. Salud Pública , 32 (1), 16-25.

Congreso de la república de Colombia. (1993). Por la cual se crea el sistema de seguridad social integral y se dictan otras disposiciones. Congreso de la República de Colombia.

Congreso de la República de Colombia. (2004). Evaluación del Sistema General de Seguridad Social en Salud y del Sistema General de Riesgos Profesionales durante los últimos 10 años. República de Colombia.

García, I. (2007). La nueva gestión pública: evolución y tendencias. Presupuesto y Gasto Público, 47, 37-64.

García-Ubaque, J. C., García-Ubaque, C., \& Benítez, L. F. (2012). Reforma colombiana en salud: propuesta de ajuste en el esquema de aseguramiento y financiación. Rev. Sal. Pública, 14 (5), 878-890.

Gorman, B. S., \& Primavera, L. H. (1983). The Complementary Use of Cluster and Factor Analysis Methods. The Journal of Experimental Education, 51(4), 165-168. http://www.jstor.org/stable/20151504

Huang, Y.-H., Ma, L., Sabljak, L. A., \& Puhala, Z. A. (2018). Development of sustainable community paramedicine programmes: a case study in Pennsylvania. Emergency Medicine Journal, 35 (6), 372-378.

IBM Corp. (2015). IBM SPSS Statistics for Windows, Version 23.0. Armonk, NY.
Jiménez Sánchez, J. I., Rojas Restrepo, F. S., \& Ramírez Tapias, D. A. (2017). Herramienta "Gerencia del Valor" para el diagnóstico financiero en las Empresas Sociales del Estado. Revista ESPACIOS, 38 (23). 1-11.

Kutzin, J. (2013). Health financing for universal coverage and health system. Bull World Health Organ., 91 (8) 602-611.

Kuwaititi, A. A., \& Al Muhanna, F. A. (2020). Challenges of privatizing academic medical centers in Saudi Arabia and appropriate strategies for implementation. International Journal of Health Governance, 68-77

Mclntyre, D., \& Kutzin, J. (2016). Health financing country diagnostic: a foundation for national strategy development. WHO.

Morales, L., \& García, J. (2017). Gestión de la crisis del sistema de salud en Bogotá. Rev Salud Pública, 19, (1), 129-133.

Ochoa, S. A., \& Moctezuma, J. (2012). Revisión crítica de la literatura sobre el análisis financiero de las empresas. Nóesis. Revista de Ciencias Sociales y Humanidades, 21 (41), 74-99.

Odoch, W. D., Senkubuge, F., \& Hongoro, C. (2021). How has sustainable development goals declaration influenced health financing reforms for universal health coverage at the country level? A scoping review of literature. Globalization and Health, 17 (59), 1-13.

Rivera, A. (2011). Coyuntura del sistema de salud en Colombia: caso Comfandi-SOS. Informe de pasantía. Universidad de San Buenaventura.

Rodríguez, J. (2012). Algunas causas de la crisis financiera en el sistema de salud en Colombia en el 2009. Econógrafos, (30), 6-23

Rodríguez, J. (2012). Vigilar y castigar: consideraciones sobre el papel de la superintendencia nacional de salud colombiana. FCE-Universidad Nacional. 
Rojas, F. (2016). Marco de referencia de la Contabilidad Pública Gubernamental: Diagnóstico del cumplimiento de los planes de saneamiento fiscal, financiero y contable de los municipios priorizados por el departamento de Antioquia. (66), Contaduría, 161-174.

Samaja, J. (2012). Epistemología y metodología. Elementos para una teoría de la investigación científica. Eudeba.

Santos, A., García, M., \& Chicaíza, L. (2016). ¿Crisis financiera o de gestión? Evolución del sector salud desde la perspectiva de los entes territoriales. . Apuntes del CENES, 61, (35), 177-206.

Suárez-Rozo, L. F., Puerto-García, S., \& Rodríguez-Moreno, L. M. (2017). La crisis del sistema de salud colombiano: una aproximación desde la legitimidad y la regulación. Revista en Gerencia y Política en Salud, 16 (32), 34-50. 